\title{
Radiopacity evaluation of composite restorative resins and bonding agents using digital and film $x$-ray systems
}

\author{
Bengi Oztas ${ }^{1}$ \\ Sebnem Kursun' \\ Gul Dinc ${ }^{2}$ \\ Kivanc Kamburoglu'
}

\begin{abstract}
Objective: The purpose of this in vitro study was to explore the radiopacity of composite resins and bonding materials using film and phosphor plates.

Methods: Nine composite dental resin specimens and human tooth slices were exposed together with an aluminium stepwedge using dental film and phosphor plates. Eight dentin bonding specimens were prepared and exposed in a similar manner. Their radiopacity on film was assessed using a transmission densitometer, and the radiopacity with phosphor plates was assessed digitally using the system's own software (Digora). Data were analysed using one-way analysis of variance (ANOVA) and post-hoc Tukey tests $(\mathrm{P}<.05)$. Film and phosphor plate radiopacity values were compared using simple regression analysis.

Results: Excellent linear correlation was found between film and phosphor plates for both composite resins and bonding agents. The composite materials Spectrum Tph and Natural Look exhibited the highest radiopacity with film and with phosphor plates, respectively. All the dentin bonding agents tested exhibited lower radiopacity than dentin.

Conclusion: Synergy, Ice, Filtek Silorane, Filtek Z250, Clearfil Majesty Posterior, Herculite Classic, Spectrum Tph, and Natural Look composite materials exhibited greater radiopacity than dentin, and all the dentin bonding agents tested exhibited lower radiopacity than either enamel or dentin. (Eur J Dent 2012;6:115-122)
\end{abstract}

Key words: Composite resin; dental bonding; densitometry; radiography

\footnotetext{
1 Department of Dentomaxillofacial Radiology, Faculty of Dentistry, Ankara University, Ankara, TURKIYE

2 Department of Restorative Dentistry, Faculty of Dentistry, Ankara University, Ankara, TURKIYE

- Corresponding author: Dr. Sebnem Kursun Ankara Universitesi, Dishekimligi Fakultesi, Agiz Dis ve Cene Radyolojisi AD. Besevler, Ankara, TURKIYE Tel: +90 3122965634

Fax: +903122123954

Email: sebnemkursundyahoo.com.tr
}

\section{INTRODUCTION}

The radiopacity of both composite resins and bonding agents is an important factor in the accurate diagnosis of secondary caries below restorative materials. Several restorative composite dental resins and dental bonding agents are available for commercial use. While no criteria exist regarding the standard radiopacity of dental bonding agents, the International Standards Organisation 
(ISO) guidelines require the radiopacity of composite materials to be equal to or greater than that of the same thickness of aluminum (ISO Standard 4049). ${ }^{1}$

In addition to their biological, physical, and mechanical properties, the radiopacity of composite resins and adhesives should be considered in selecting the most suitable material for specific clinical situations. Composite resin radiopacity can affect the ability to detect voids and overhangs and to distinguish normal tooth structure from restorative material. ${ }^{2-13}$ Furthermore, in addition to other factors that influence the detectability of recurrent caries (proximity of the lesion to the adjacent restoration, size of the initial lesion, geometry of the radiographic projection and orientation of the lesion), ${ }^{14}$ the use of a restorative material with a radiopacity similar to or slightly greater than that of enamel can improve the radiographic detection of caries..$^{3-5,9-13}$ Radiopacity is usually expressed in terms of aluminum thickness, ${ }^{2-6,9-13,15}$ and many researchers use aluminum stepwedges to compare the radiopacity of restorative materials under typical radiographic conditions. Radiopacity can be measured by transmission densitometry, direct digital radiography, or indirect digital radiography. In transmission densitometry, optical density is calculated as a logarithmic measure of the ratio of light transmitted through a film image to incident light. Digital image analysis, on the other hand, provides a direct record of radiographic density as determined by pixel shade, which is automatically recorded by computer software as a value between 0 and 255. ${ }^{11}$ Direct digital radiography employs one of two types of image sensors: a charge-coupled device (CCD), which provides a direct electronic reading, or phosphor plates, which store image information and emit light on demand that is measured by a separate sensor. ${ }^{8,16}$ Indirect digital imaging involves digitization of chemically processed radiographs and the use of specific software to identify grey values. ${ }^{4,15}$ Direct digitization offers several advantages, including immediate image capture without the need for processing chemicals, a wide dynamic range, and high sensitivity to radiation exposure. ${ }^{17}$ Direct digital imaging also eliminates the loss of information that may occur with indirect digitization. ${ }^{11}$

The purpose of this in vitro study was to record and compare the radiopacity of composite resins and dental bonding agents using film and phosphor plates.

\section{MATERIALS AND METHODS}

Sample Preparation:

Composite Materials;

Nine resin-based restorative composite materials were used in this study: Synergy (Coltene, Altstaeten SG, Switzerland), Ice (SDI, Bayswater, Victoria, Australia), Filtek Silorane (3M, St Paul, MN, USA), Aelite Aesthetic Enamel (Bisco, Inc, Itasca, IL, USA), Filtek Z250 (3M, St Paul, MN, USA), Clearfil Majesty Posterior (Kuraray, Osaka, Japan), Herculite Classic (Kerr/Sybron, Orange, CA, USA), Spectrum Tph (Dentsply DeTrey, Konstanz, Germany), Natural Look (DFL, Rio de Janeiro, Brazil). Their specifications are given in Table 1.

Composite specimens were prepared in triplicate for each material tested using steel ring moulds with an internal diameter of $10 \mathrm{~mm}$ and a height of $1 \mathrm{~mm}$. Moulds were placed between two glass plates, clamped under pressure, and lightcured (Hilux Dental Curing, Benlioglu Dental Inc, Ankara, Turkeyl according to the manufacturers'

Table 1. Composite resin materials evaluated in the study.

\begin{tabular}{lccc}
\hline Product & Manufacturer & Filler \%(wt/vol) & Type \\
\hline Synergy & Coltene & $77 / 58$ & Nanohybrid \\
Ice & SDI & NA/61 & Nanohybrid \\
Filtek Silorane & 3 M Espe & $76 / 55$ & Microhybrid \\
Aelite Aesthetic Enamel & Bisco & $73 / 54$ & Nanohybrid \\
Filtek Z250 & 3M Espe & $77.4 / 60$ & Hybrid \\
Clearfil Majesty & Kuraray & $92 / 82$ & Nanohybrid \\
Herculite Classic & Kerr & $78 /$ NA & Hybrid \\
Tph Spectrum & Dentsply & $77 / 57$ & Hybrid \\
Natural Look & DFL & $77 / 58.5$ & Microhybrid \\
\hline
\end{tabular}

NA, not available; wt, weight; vol, volume 
instructions. In addition to composite samples, 1-mm enamel and dentin samples were prepared from freshly extracted premolar teeth.

\section{Bonding Materials;}

Eight dentin bonding agents were used in this study: Optibond Solo Plus (Kerr/Sybron, Orange, CA, USA), Prime \& Bond NT (Dentsply DeTrey, Konstanz, Germany), Adper Single Bond (3M, St Paul, MN, USA), Go (SDI, Bayswater, Victoria, Australia), Filtek Silorane Bond and Primer (3M, St Paul, MN, USA), Clearfil SE Bond (Kuraray, Osaka, Japan) Xeno V (Dentsply DeTrey, Konstanz, Germany), Meta P\&Bond (Metabiomed, Chungbuk, Korea). Their specifications are given in Table 2.

Bonding agent specimens were prepared in triplicate for each material tested using orthodontic elastic rubber bands $3 \mathrm{~mm}$ in diameter and $1 \mathrm{~mm}$ high. An applicator was used to apply the bonding agent inside the rubber band, which was placed between two glass plates and light-cured (Hilux Dental Curing, Benlioglu Dental Inc, Ankara, Turkeyl according to the manufacturer's instruction. The process was repeated until a specimen of the required size was obtained. In addition to bonding agent samples, 1 -mm enamel and dentin samples were prepared from freshly extracted premolar teeth.

Image Exposure:

Film;

Each specimen was placed on occlusal film together with an aluminum stepwedge $199.5 \%$ purity, $1 \mathrm{~mm}$ per step) to establish radiopacity equivalents for the resin composites, bonding agents, dentin and enamel. Composites were imaged together with a 12-step wedge using Kodak Ultraspeed DF 50, Size 4 (Eastman Kodak Company, Rochester, NY, USA), and bonding agents were imaged to- gether with a 7-step wedge using Kodak D Speed Dental Film, Size 2 (Eastman Kodak). Films were exposed using a dental $x$-ray machine (Evolution X 3000-2C, New Life Radiology Srl, Italyl at $70 \mathrm{kVp}$ and $8 \mathrm{~mA}$. Composites were exposed for $0.35 \mathrm{~s}$ with a $35-\mathrm{cm}$ film-target distance, and bonding agents were exposed for $0.18 \mathrm{~s}$ with a $23.5-\mathrm{cm}$ film-target distance.

Films were processed using an automatic processor (Extra-x Velopex, Medivance Instruments Limited, London, England) with fresh solution (Hacettepe, Ankara, Turkey) mixed according to the manufacturer's instructions (Figure 1a, 1b).The base-plus-fog density was obtained by processing an unexposed occlusal film in the same automatic processor. The radiographic densities of each step of the stepwedge and the samples dentin, enamel and unexposed films were measured using a densitometer (Macbeth TD 932, Newburgh, NY, USA) with a $0.1-\mathrm{mm}$ aperture. Three readings were taken for each film, and the mean was calculated. A graph of the optical density values for the entire stepwedge was plotted and used to determine the aluminum thickness equivalent values of the composites, bonding agents, enamel and dentin.

\section{Direct Digitization;}

Digital images of resin composites and bonding agents were recorded with a Digora Direct Digital system (Soredex, Helsinki, Finland) using a Size 3 plate for the composites and a Size 2 plate for the bonding agents. Phosphor plates were exposed using the same process for each material described above. To avoid possible differences related to the phosphor plates, the same plates were used for all specimens. Following exposure, the images were transferred to a computer using a Digora scanner and stored in the tagged image file (TIF) format. Images were viewed using Digora

Table 2. Bonding adhesive materials evaluated in the study.

\begin{tabular}{lcc}
\hline Adhesive systems & Manufacturer & Total-etch/Self-etch \\
\hline Optibond Solo Plus & KERR & Total etch \\
\hline Prime\&Bond NT & DENTSPLY & Total-etch \\
\hline Adper Single Bond & $3 M$ ESPE & Total-etch \\
Go & SDI & Self-etch \\
Silorane Primer and Bond & 3M ESPE & Self-etch \\
Clearfil SE Bond & KURARAY & Self-etch \\
Xeno V & DENTSPLY & Self-etch \\
Meta P\&Bond & METABIOMED & Total-etch \\
\hline
\end{tabular}


for Windows 5.1 on a 15" 32-bit color monitor at a $1024 \times 768$ pixel resolution (256 grey levels). The radiodensities of the samples were measured using the software provided by the manufacturer. Five measurements were taken per sample lone point in the central area and one point in each quadrant), and the means were calculated. A calibration curve was generated using the grey pixel values of the stepwedge steps, and the resulting graph was used to establish aluminum equivalent values of the composites, bonding agents, enamel and dentin.

The mean radiopacity values for each group of materials tested were compared using one-way analysis of variance (ANOVA) and post-hoc Tukey HSD tests $(P<0.05)$. The mean radiopacity values for each group were compared with those of enamel and dentin using a means test, and simple regression analysis was used to compare the results of the two radiographic methods used.

\section{RESULTS}

The aluminum equivalent thicknesses of the resin composites and bonding agents indicated a high correlation between the radiopacity of composite resins ( $r=.981)$ and bonding agents ( $r=.968$ ) as measured using film and a direct digital system.

\section{Transmission Densitometry:}

Figure 2 shows the aluminum equivalent values of the composite resins, enamel and dentin. The transmission densitometry optical density values of the composite resins ranged from 0.86 to 1.26 (Table 3). Dentsply Spectrum Tph exhibited the highest radiopacity of the composites tested, and Bisco Aelite exhibited the lowest. No significant differences were found among Coltene Synergy, 3M Filtek Z250, and Kerr Herculite ( $P>.05)$ or between SDI Ice and Kuraray Majesty (P>.05).

Figure 3 shows the aluminum equivalent values for the dental bonding agents, enamel, and dentin. The transmission densitometry optical density values of the bonding agents ranged from 1.28 to 1.35 (Table 4). Among the tested bonding agents, Kerr Optibond Solo exhibited the highest radiopacity, and Metabiomed Meta P\&Bond and Dentsply Xeno $V(P>.05)$ showed the lowest.
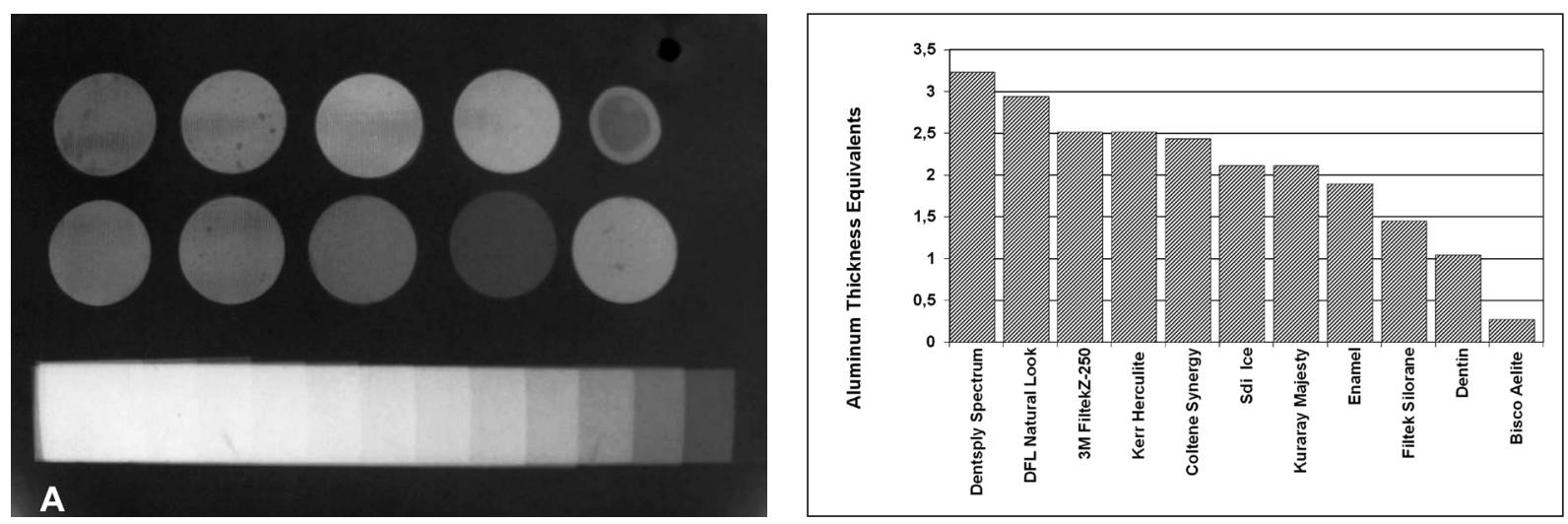

Figure 2. Aluminum thickness equivalents of resin composites and tooth substances for conventional method.
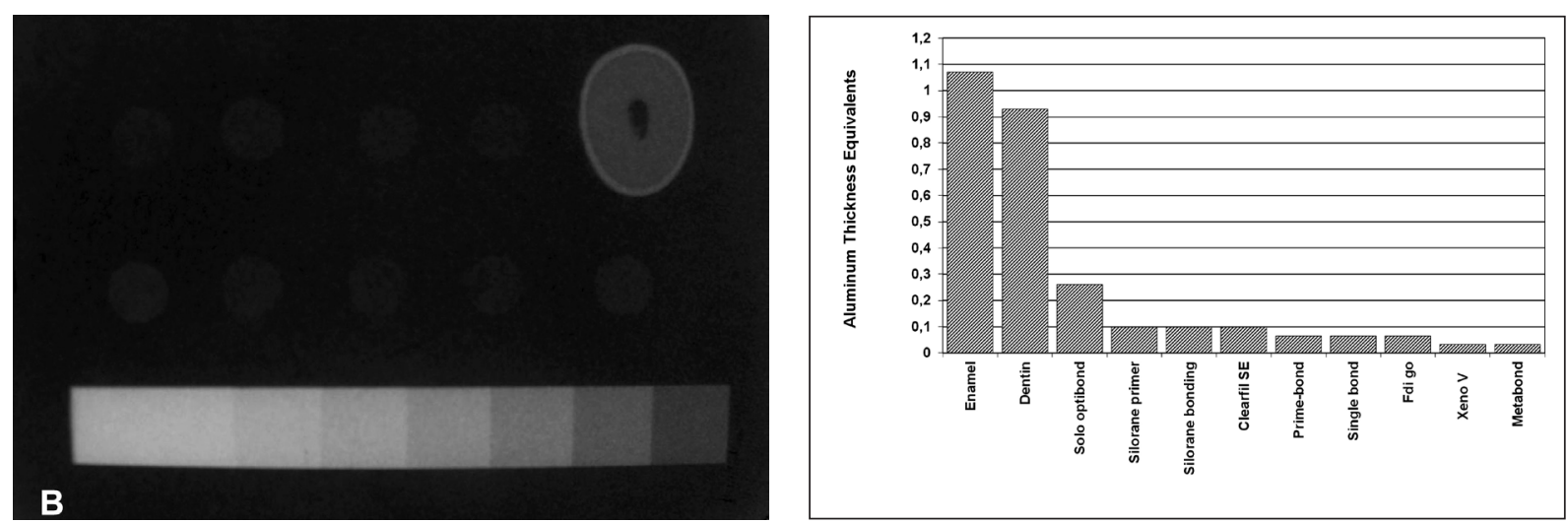

Figure 1. A) Radiograph of composite specimens, tooth, and aluminum stepwedge. B) Radiograph of bonding specimens, tooth, and aluminum stepwedge.

Figure 3. Aluminum equivalent values of bonding agents and tooth substances for conventional method. 
Digital Analysis:

Figure 4 shows the aluminum equivalent values of the composite resins, enamel, and dentin. With the digital system, the mean grey values of the composites ranged from 84 to165 (Table 5). DFL Natural Look exhibited the highest radiopacity among the composites tested, and Bisco Ael-

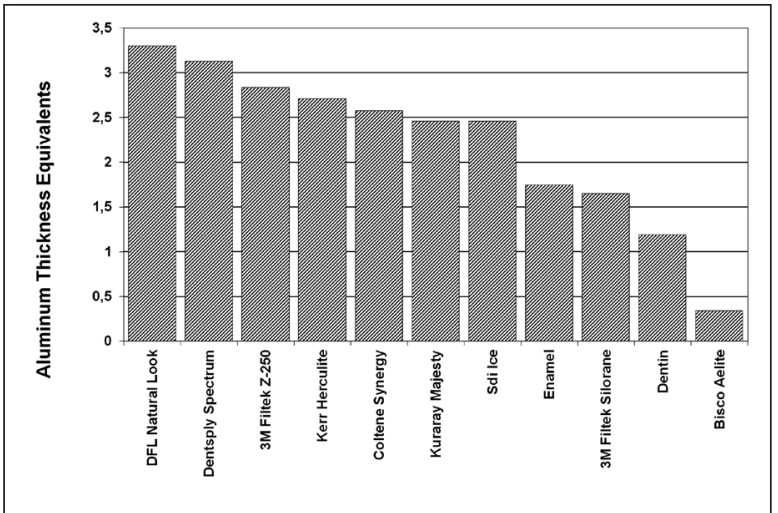

Figure 4. Aluminum equivalent values of resin composites and tooth substances for digital method. ite exhibited the lowest. No significant differences ( $P>0.05$ ) were found among Coltene Synergy, 3M Filtek Z250, SDI Ice, and Kerr Herculite or between Dentsply Spectrum and DFL Natural Look.

Figure 5 shows the aluminum equivalent values of the dental bonding agents, enamel, and dentin. With the digital system, the mean grey

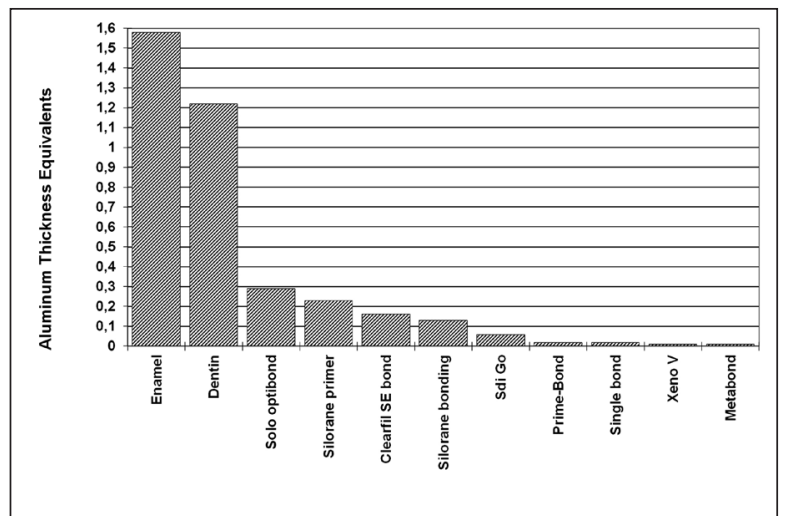

Figure 5. Aluminum equivalent values of bonding agents and tooth substances for digital method.

Table 3. Radiopacity test results of composite resins for transmission densitometry.

\begin{tabular}{llc}
\hline Material & (n) & Mean Optical Density \\
\hline Dentsply Spectrum & 3 & $0.86^{*} \pm 0.005$ \\
DFL Natural Look & 3 & $0.89 \pm 0.005$ \\
Kerr Herculite & 3 & $0.94 \pm 0.005$ \\
\hline 3M Filtek Z-250 & 3 & $0.94 \pm 0.01$ \\
Coltene Synergy & 3 & $0.95 \pm 0.15$ \\
SDI Ice & 3 & $0.99 \pm 0.01$ \\
Kuraray Clearfil Majesty & 3 & $0.99 \pm 0.005$ \\
Enamel & 3 & $1.02 \pm 0.005$ \\
3M Filtek Silorane & 3 & $1.08 \pm 0.011$ \\
Dentin & 3 & $1.14 \pm 0.011$ \\
Bisco Aelite & 3 & $1.26 \pm 0.01$ \\
\hline
\end{tabular}

*Mean value and standard deviation of radiographic densities

Table 4. Radiopacity test results of bonding materials for transmission densitometry.

\begin{tabular}{llc}
\hline Material & (n) & Mean Optical Density \\
\hline Enamel & 3 & $1.06^{*} \pm 0.005$ \\
Dentin & 3 & $1.11 \pm 0.005$ \\
Kerr Solo & 3 & $1.28 \pm 0.005$ \\
3M Silorane Primer & 3 & $1.33 \pm 0.005$ \\
3M Silorane Bond & 3 & $1.33 \pm 0.005$ \\
Kuraray Clearfil SE & 3 & $1.33 \pm 0.005$ \\
Dentsply Prime\&Bond & 3 & $1.34 \pm 0.005$ \\
3M Adper Single & 3 & $1.34 \pm 0.005$ \\
SDI Go & 3 & $1.34 \pm 0.005$ \\
\hline Dentsply Xeno V & 3 & $1.35 \pm 0.005$ \\
Metabiomed MetaP\&Bond & 3 & $1.35 \pm 0.005$ \\
\hline
\end{tabular}

* Mean value and standard deviation of radiographic densities 
values of the bonding agents ranged from 84 to 170 (Table 6). Kerr Optibond Solo exhibited the highest radiopacity of the bonding agents tested, and Metabiomed Meta P\&Bond exhibited the lowest. No significant differences ( $P>$.05) were found among Dentsply Prime \& Bond NT, 3M Single Bond, SDI Go, Dentsply Xeno V, and Metabiomed Meta P\&Bond.

\section{DISCUSSION}

The radiopacity of composite resins and dentin bonding agents plays a critical role in the ability to distinguish composite restorations from both tooth structure and secondary caries on a radiograph. Currently, no criteria exist regarding bonding agent radiopacity, and while the ISO recommends that composite resin radiopacity exceed that of dentin and equal or exceed that of the same thickness of aluminum (ISO Standard 4049), no definitive upper limit has been established. However, excessive radiopacity has been reported to be a negative characteristic that may reduce the ability to diagnose recurrent caries and other defects. ${ }^{4,9-11}$

In the present study, for both film and phosphor plates, two of the nine composite materials (Bisco Aelite and 3M Filtek Silorane) had radiopacity values lower than that of enamel, and Bisco Aelite had a radiopacity value lower than that of the same thickness $(1 \mathrm{~mm})$ of aluminum. In line with earlier studies, the present study found composite resins (Dentsply Spectrum Tph, 3M Filtek Z250, Kerr Herculite) that exhibited higher opacity than enamel.,9-11 In some cases, differences were observed in the mean radiopacity values of the same materials reported in this study and previous studies; ${ }^{9,12}$ these differences may be attributed to variations in testing conditions (film speed, exposure time, voltage, age of developing/fixing solutions).

In the present study, all the bonding agents exhibited less radiopacity than enamel. Although

Table 5. Radiopacity test results of composite resins for digital system.

\begin{tabular}{llc}
\hline Material & (n) & Mean gray value \\
\hline DFL Natural Look & 3 & $165.3^{*} \pm 4.1$ \\
\hline Dentsply Spectrum & 3 & $160.6 \pm 4.5$ \\
3M Filtek Z-250 & 3 & $152.3 \pm 4.7$ \\
\hline Herculite Kerr & 3 & $147 \pm 3.6$ \\
Coltene Synergy & 3 & $143.3 \pm 8.7$ \\
SDI Ice & 3 & $139.3 \pm 5.1$ \\
\hline Kuraray Clearfil Majesty & 3 & $126.6 \pm 7.7$ \\
\hline Enamel & 3 & $118 \pm 4$ \\
3M Filtek Silorane & 3 & $115.3 \pm 5.5$ \\
Dentin & 3 & $101.6 \pm 2.5$ \\
\hline Bisco Aelite & 3 & $84 \pm 9.5$ \\
\hline
\end{tabular}

* Mean value and standard deviation of radiographic densities

Table 6. Radiopacity test results of bonding materials for digital system.

\begin{tabular}{llc}
\hline Material & (n) & Mean gray value \\
\hline Enamel & 3 & $170.3^{*} \pm 2.3$ \\
\hline Dentin & 3 & $158.6 \pm 6.0$ \\
Kerr Solo & 3 & $109.6 \pm 2.0$ \\
\hline 3M Silorane Primer & 3 & $105.0 \pm 3.6$ \\
\hline Kuraray Clearfil SE & 3 & $100.0 \pm 2.0$ \\
3M Silorane Bond & 3 & $96.6 \pm 1.1$ \\
\hline SDI Go & 3 & $90.0 \pm 1.0$ \\
\hline 3M Adper Single & 3 & $89.0 \pm 5.2$ \\
\hline Dentsply Prime\&Bond & 3 & $86.6 \pm 2.3$ \\
\hline Metabiomed MetaP\&Bond & 3 & $84.0 \pm 3.0$ \\
\hline Dentsply Xeno V & 3 & $83.6 \pm 3.2$ \\
\hline
\end{tabular}

* Mean value and standard deviation of radiographic densities 
bonding agent radiopacity can influence the accurate diagnosis of recurrent caries beneath restorations, the literature contains only one previous study on the radiopacity of bonding agents. In that study, Hotta and Yamamoto ${ }^{13}$ compared the radiopacity of 15 bonding agents. Optibond Solo Plus and Adper Single Bond are common to the present study. They found that with the exception of Fluoro Bond II (Shofu, Kyoto, Japan), which had an aluminum equivalent radiopacity value of $0.918 \mathrm{~mm}$, all other bonding agents tested had radiopacity values below that of dentin. Both Hotta and Yamamoto $^{13}$ and the present study found that Optibond Solo Plus exhibited higher opacity than Adper Single Bond.

The present study measured radiopacity using both a transmission densitometer and a direct digital system. Transmission or photographic densitometry and indirect digital image analysis are the most widely used techniques for evaluating the radiopacity of dental materials. $3,5,6,9,10,12-14$ However, some studies, 3,7,11,18 including ours, have used direct digital image analysis, which eliminates the problem of loss of information that may occur with the use of indirect digital imaging. ${ }^{11}$ Despite a few discrepancies, our study found a linear correlation between conventional film and the Digora digital system in the measurement of composite resin and bonding agent radiopacity. These results are in line with those of Sabbagh et $\mathrm{al}^{3}{ }^{3}$ but conflict with those of Wenzel et al, ${ }^{11}$ who reported digital systems to be less reliable than conventional film. Another study that examined the radiopacity of resin composites using CCDs, the Digora storage phosphor system, and Extraspeed plus film found that imaging modality was unlikely to affect radiopacity values. ${ }^{19}$

Any discrepancies between the two methods may be attributed to the differences between analogue and digital technologies. ${ }^{4}$ Whereas conventional analogue film uses an $\mathrm{x}$-ray beam to record the density of the silver grains in photographic emulsion, ${ }^{4,20}$ which results in a continuous density curve, ${ }^{4,10}$ digital systems provide direct measurements of grey shades in absolute numbers (from 0 to 255).

In conclusion, in line with previous studies, our study found that the majority of composite materials tested complied with the ISO-4049 standard. However, all the bonding agents tested had ra- diopacity values lower than that of dentin, which could cause confusion in the diagnosis of secondary caries by mimicking dental caries. Further studies should be conducted with commercially available adhesive materials to encourage their manufacturers to produce materials with more appropriate opacity levels.

\section{REFERENCES}

1. International Standard 4049 Geneva International Organization for Standardization Dentistry- Polymer based fillings, restorative and luting materials,2000.

2. Cook WD. An investigation of the radiopacity of composite restorative materials. Aust Dent J 1981;26:105-112.

3. Murchison DF, Charlton DG, Moore WS. Comparative radiopacity of flowable resin composites. Quintessence Int 1999;30:179-184.

4. Sabbagh J, Vreven J, Leloup G.Radiopacity of resin - based materials measured in film radiographs and storage phospor plate (digora). Oper Dent 2004;29:677-684.

5. Gurdal P, Akdeniz BG. Comparison of two methods for radiometric evaluation of resin-based restorative materials. Dentomaxillofac Radiol 1998;27:236-239.

6. Toyooka H, Taira M, Wakasa K, Yamaki M, Fujita M, Wada T. Radiopacity of 12 visible-light-cured dental composite resins. J Oral Rehabil 1993;20:615-622.

7. Carlos JS, Santana FR, Fonseca RB, Martins LRM, Neto FH. In vitro analysis of the radiodensity of indirect composites and ceramic inlay systems and its influence on the detection of cement overhangs. Clin Oral Invest 2007;11:331-336.

8. Nomoto R, Mishima A, Kobayashi K, McCabe JF, Darvell BW, Watts DC, Momoi Y, Hirano S. Quantitive determination of radio-opacity:equivalence of digital and film x-ray systems. Dent Mater 2008;24:141-147.

9. Turgut MD, Attar N, Önen A. Radiopacity of direct esthetic restorative materials. Oper Dent 2003;28:508-514.

10. Curtis PM, Fraunhofer A, Farman AG. The radiographic density of composite restorative resins. Oral Surg Oral Med Oral Pathol Oral Radiol Endod 1990;70:226-230.

11. Salzedas LMP, Louzada MJQ, Oliveira Filho AB. Radiopacity of restorative materials using digital images. J Appl Oral Sci 2006; 14:147-152.

12. Marouf N, Sidhu SK. A study on the radiopacity of different shades of resin-modified glass-ionomer restorative materials. Oper Dent 1998;23:10-14.

13. Hotta M, Yamamoto K. Comparative radiopacity of bonding agents. J Adhes Dent 2009;11:207-212. 
14. Nair MK, Ludlow JB, May KN, Nair UP, Johnson MP, Close JM. Diagnostic accuracy of intraoral film and direct digital images for detection of simulated recurrent decay. Oper Dent 2001;26:223-230

15. Carvalho-Junior JR, Correr-Sobrinho L, Correr AB, Sinhoreti MAC, Consani S, Sausa-Neto MD. Radiopacity of root filling materials using digital radiography. Int Endod $J$ 2007;40:514-520.

16. Grondahl H-G, Wenzel A, Borg E, Tammisalo E. An image plate system for digital intra-oral radiography. Dent Update $1996 ; 23: 334-337$.

17. Wenzel A, Hintze $H$, Horsted-Bindslev P. Discrimination between restorative dental materials by their radiopacity measured in film radiographs and digital images. J Forensic Odontostomatol 1998;16:8-13.

18. Gu S, Rasimick BJ, Deutsch AS, Musikant BL. Radiopacity of dental materials using a digital x-ray system. Dent Mater 2006;22:765-770.

19. Farman TT, Farman AG, Scarfe WC, Goldsmith LJ. Optical densities of dental resin composites:a comparison of CCD, storage phosphor and ektaspeed plus radiographic film. Gen Dent 1996;44:532-537.

20. van Der Stelt PF. Principles of digital imaging. Dent Clin $N$ Am 2000;44:237-248. 